\title{
Combination of peroxisome proliferator-activated receptor $\alpha / \gamma$ agonists may benefit type 2 diabetes patients with coronary artery disease through inhibition of inflammatory cytokine secretion
}

\author{
JINRU WEI, QUAN TANG, LIJUAN LIU and JIANBIN BIN \\ Department of Cardiology, The Fifth Affiliated Hospital of Guangxi Medical University, \\ Liuzhou, Guangxi 545006, P.R. China
}

Received October 11, 2012; Accepted December 13, 2012

DOI: $10.3892 /$ etm.2013.891

\begin{abstract}
Patients with type 2 diabetes mellitus (T2DM) have a higher risk of cardiovascular disease (CVD). Peroxisome proliferator-activated receptors (PPARs) play an important role in the regulation of energy homeostasis. Therefore, we aimed to observe the effects of combined PPAR $\alpha / \gamma$ agonists on T2DM patients with coronary artery disease (CAD). Patients were randomly divided into a rosiglitazone (RSG) group ( $n=20)$, a bezafibrate (BEZ) group $(n=20)$, a combination of RSG and BEZ group $(n=20)$ and a control group $(n=20)$. Plasma C-reactive protein (CRP) and monocyte chemoattractant protein-1 (MCP-1) were measured by enzyme-linked immunosorbent assay before and 12 weeks after treatment. Fasting blood glucose (FBG), fasting insulin, insulin resistance index (IRI), hemoglobin A1c (HbA1c), lipid levels and body mass index were also investigated. At the end of the treatment, FBG, insulin, IRI, HbA1c and triglyceride levels decreased and the level of high-density lipoprotein cholesterol increased in the RSG, BEZ and combination groups. A decrease in low-density lipoprotein cholesterol was only observed in the combination group. Although the total cholesterol levels in all groups decreased, no significant difference was noted. The levels of CRP and MCP-1 were reduced in patients in the RSG, BEZ and combination groups. In addition, RSG, BEZ and the combination of RSG and BEZ also inhibited MCP-1 secretion. The combination of RSG and BEZ was more efficient than RSG or BEZ alone in downregulating cytokines. In conclusion, our results suggest that a combination of RSG and BEZ may be more efficient than RSG or BEZ alone in the treatment of T2DM patients with CAD.
\end{abstract}

Correspondence to: Dr Jinru Wei, Department of Cardiology, The Fifth Affiliated Hospital of Guangxi Medical University, 8 Wenchang Road, Liuzhou, Guangxi 545006, P.R. China E-mail: docweijinru@126.com

Key words: diabetes mellitus, coronary heart disease, peroxisome proliferator-activated receptor $\alpha$, peroxisome proliferator-activated receptor $\gamma$, inflammatory cytokine

\section{Introduction}

Patients with type 2 diabetes mellitus (T2DM) have a higher risk of cardiovascular disease (CVD), including coronary artery disease (CAD), the main cause of premature morbidity and mortality in these patients $(1,2)$. Inflammation has been indicated to be an important contributor to the development of T2DM and CAD, not only by promoting atherogenesis, but also by inducing insulin resistance (IR) and $\beta$-cell impairment.

Peroxisome proliferator-activated receptors (PPARs) belong to the nuclear receptor superfamily and are ligand-activated transcription factors. There are three isoforms, $\alpha, \beta$ and $\gamma$ (3). PPARs play an significant role in the regulation of energy homeostasis by regulating the expression of a variety of genes involved in lipid and carbohydrate metabolism (4). Data from murine models also suggest that PPAR agonists have independent anti-atherosclerotic actions, including the suppression of vascular inflammation, oxidative stress and activation of the renin-angiotensin system (RAS) (5). PPAR agonists reduce the production of tumor necrosis factor (TNF)- $\alpha$, interleukin (IL)-6 and IL-1 $\beta$ (6,7). PPAR $\alpha$ activation indirectly modulates inflammatory components in high-density lipoprotein (HDL), including apolipoprotein A1 (apoA1), serum amyloid A and paraoxonase-1 (8). PPAR $\gamma$ activators inhibit the expression of matrix metalloproteinase (MMP)-9 in human vascular smooth muscle cells and macrophages (9). PPAR $\gamma$ activators also inhibit the production of TNF- $\alpha$, IL- 6 and IL-1 $\beta$ by activated monocytes (10). A number of these effects are mediated by transrepression of the nuclear factor $-\kappa \mathrm{B}(\mathrm{NF}-\kappa \mathrm{B})$ and activator protein-1 (AP-1) dependent pathways $(7,11)$.

The dual PPAR $\alpha / \gamma$ agonists were developed due to the apparent efficacy of PPAR $\alpha$ and PPAR $\gamma$ agonists individually on metabolic control, providing the possibility of optimizing the metabolic and anti-atherosclerotic actions through activation of the two receptors. These dual agonists, including ragaglitazar and muraglitazar, demonstrate a higher affinity for PPAR $\gamma$ than conventional thiazolidinediones and are highly effective at improving metabolic parameters $(12,13)$. However, the effects of these agents on atherogenesis are not clearly understood. Treatment with a dual PPAR $\alpha / \gamma$ agonist, compound $3 \mathrm{q}$, notably increased atherosclerosis in control apoE knockout mice (14), while PPAR $\gamma$ and $\alpha$ agonists used 
alone in this model were protective $(15,16)$. Furthermore, treatment with a dual PPAR agonist in mice resulted in plaque accumulation accompanied by an increase in aortic gene expression of the pro-inflammatory molecules, P-selectin, CD36, vascular cell adhesion molecule 1 (VCAM-1) and monocyte chemoattractant protein-1 (MCP-1) and increased macrophage infiltration, an effect not observed with the single PPAR agonists, rosiglitazone (RSG) or gemfibrozil (14). One study suggested that dual PPAR $\alpha / \gamma$ agonists are also associated with an increased risk of adverse cardiovascular events when used by individuals with diabetes (17). Therefore, there is a long distance from the bench to the clinic for the dual PPAR $\alpha / \gamma$ agonists and a new strategy that may benefit T2DM patients with CAD should be explored.

RSG, a PPAR $\gamma$ agonist typically used to treat T2DM patients $(18,19)$, significantly reduces homocysteine-induced reactive oxygen species and the secretion of MCP-1 and IL-8 in human monocytes. It also significantly decreases plasma C-reactive protein (CRP) and MCP-1 levels in T2DM patients with CAD (20). Bezafibrate (BEZ), a PPAR $\alpha$ agonist and an effective drug in the treatment of dyslipidemia, has been shown to be effective in the primary prevention of cardiovascular events (21). Therefore, we combined these PPAR $\alpha$ and PPAR $\gamma$ agonists in the treatment of T2DM patients with CAD, with the expectation of greater efficacy and other advantages.

\section{Materials and methods}

Subjects. Eighty T2DM patients with CAD were enrolled from the Department of Cardiovascular, The Fifth Affiliated Hospital of Guangxi Medical University, China, from January 2006 to December 2008. T2DM was defined as the level of glucose, including fasting blood glucose (FBG) $\geq 7.0 \mathrm{mmol} / 1$, casual plasma glucose $\geq 11.1 \mathrm{mmol} / 1$ or plasma glucose $\geq 11.1 \mathrm{mmol} / \mathrm{l}, 2 \mathrm{~h}$ after an oral glucose tolerance test (OGTT).

T2DM patients with CAD, including angina pectoris and myocardial infarction, were diagnosed according to the criteria set out in the American College of Cardiology/ American Heart Association (ACC/AHA) guidelines (22). Coronary angiography confirmed at least one stenosis $>50 \%$. The exclusion criteria were: complications with infectious or inflammatory diseases or impaired liver or kidney function; coexisting heart failure [New York Heart Association (NYHA) class III-IV]; presence of a malignant tumor; hematological diseases; or treatment with protamine zinc insulin or isophane insulin.

Study design. The patients were randomly assigned to an RSG group (4 mg/day, n=20), a BEZ group (400 mg/day, n=20), a combination group (RSG plus BEZ, $n=20$ ) or a control group (conventional therapy, $\mathrm{n}=20$ ). All patients received conventional therapy (including oral hypoglycemic agents or subcutaneous insulin injection and low dose statins) for DM and CAD for 12 weeks. RSG was purchased from GlaxoSmithKline (Tianjing, China) and BEZ was purchased from Tianlishidiyi, Ltd. (Huaian, China). Inflammatory and metabolic factors were tested before treatment and after 12 weeks in fasting venous blood. This study was approved by the ethics committee of the Fifth Affiliated Hospital of Guangxi Medical University. All subjects gave their written informed consent.
Blood parameter analysis. Before treatment and at 12 weeks after treatment, venous blood samples were collected and centrifuged immediately at 3,000 rpm for $20 \mathrm{~min}$. The supernatant was collected and stored at $-70^{\circ} \mathrm{C}$. Plasma CRP and MCP-1 were determined by enzyme-linked immunosorbent assay (ELISA; R\&D Systems, Minneapolis, MN, USA) according to the manufacturer's instructions. FBG was measured by the standard oxidase method and lipids were measured by the standard dry chemistry method. Hemoglobin A1c (HbAlc) was measured by high performance liquid chromatography (HPLC). Insulin was determined by chemiluminescence. The estimate of IR by homeostasis model assessment (HOMA-IR) was as follows: IR = [fasting insulin (Fins; IU/ml) - fasting glucose (mmol/l)] /22.5 (23).

Responsiveness of monocytes to lipopolysaccharide. Twelve weeks after treatment, venous blood samples were obtained from fasting subjects to evaluate the effect of RSG and BEZ on MCP-1 production induced by low-dose lipopolysaccharide (LPS) in isolated monocytes. Whole blood was separated into peripheral blood mononuclear cells (PBMCs) and neutrophils using NycoPrep ${ }^{\mathrm{TM}} 1.077$ (Life Technologies Co., Carlsbad, CA, USA) and then monocytes were isolated by their adherence to the flasks. Adherent cells were then detached and resuspended in RPMI-1640 medium containing $5 \%$ autologous serum. Then, monocytes $\left(5 \times 10^{5}\right)$ were incubated at $37^{\circ} \mathrm{C}$ with or without LPS $(0.01 \mu \mathrm{g} / \mathrm{ml})$ for $24 \mathrm{~h}$. The supernatant was harvested and stored at $-70^{\circ} \mathrm{C}$ for further MCP-1 examination.

Statistical analysis. CRP and MCP-1 were expressed as the median and range and were analyzed by the Wilcoxon matched-pairs signed ranks test. The difference between groups was analyzed by the Mann-Whitney test. Age, body mass index, metabolic factors, including cholesterol, triglycerides (TGs) and glucose were expressed as the mean \pm standard deviation. A paired Student's t-test was used to compare values pre- and post-treatment. One-way analysis of variance (ANOVA) was performed for multiple comparisons followed by Dunnett's test. $\mathrm{P}<0.05$ (two-tailed) was considered to indicate a statistically significant difference.

\section{Results}

Baseline demographic characteristics. Clinical and metabolic parameters of the patients are presented in Table I. Age, gender, smoking status, hypertension, FBG, Fins, HOMA-IR, HbAlc, lipid levels and MCP-1 did not demonstrate significant differences among the four groups $(\mathrm{P}>0.05)$. The CRP levels in the BEZ and combination groups were higher than those in the RSG and control groups; however, the differences were not statistically significant $(\mathrm{P}>0.05)$.

FBG, Fins, HOMA-IR and HbAlc levels. The FBG levels in the RSG and combination groups were significantly decreased compared with those before treatment; however, no significant reduction was noted in the BEZ and control groups (Fig. 1A). Fins levels decreased after 12 weeks of treatment with RSG, BEZ or the combination of RSG and BEZ (Fig. 1B). Although a slight decrease was also observed in the control group, no 
Table I. Clinical and metabolic characteristics before treatment.

\begin{tabular}{|c|c|c|c|c|c|}
\hline Variables & RSG group & BEZ group & Combination group & Control group & P-value \\
\hline Male/female (n) & $14 / 6$ & $13 / 7$ & $12 / 8$ & $14 / 6$ & 0.535 \\
\hline Age (years) & $60.6 \pm 9.61$ & $62.8 \pm 9.41$ & $62.9 \pm 9.00$ & $60.4 \pm 8.46$ & 0.715 \\
\hline Smoking (n) & 5 & 6 & 5 & 6 & 0.275 \\
\hline Hypertension (n) & 5 & 6 & 5 & 6 & 0.230 \\
\hline $\mathrm{BMI}\left(\mathrm{kg} / \mathrm{m}^{2}\right)$ & $25.19 \pm 4.01$ & $24.79 \pm 3.50$ & $24.97 \pm 3.03$ & $24.37 \pm 2.80$ & 0.407 \\
\hline FPG (mmol/l) & $7.27 \pm 1.69$ & $7.77 \pm 4.08$ & $8.57 \pm 4.42$ & $7.45 \pm 2.86$ & 0.925 \\
\hline Fins (mU/1) & $11.48 \pm 4.65$ & $13.07 \pm 4.89$ & $12.28 \pm 4.61$ & $12.07 \pm 4.94$ & 0.868 \\
\hline HOMA-IR & $3.69 \pm 1.61$ & $4.07 \pm 2.05$ & $4.77 \pm 3.29$ & $3.74 \pm 1.56$ & 0.423 \\
\hline HbA1c (\%) & $7.84 \pm 1.58$ & $7.58 \pm 2.01$ & $8.05 \pm 2.10$ & $7.49 \pm 1.83$ & 0.582 \\
\hline $\mathrm{TC}(\mathrm{mmol} / \mathrm{l})$ & $4.73 \pm 0.92$ & $4.43 \pm 0.81$ & $4.49 \pm 0.77$ & $4.52 \pm 1.07$ & 0.541 \\
\hline TG (mmol/l) & $1.92 \pm 0.98$ & $2.06 \pm 1.25$ & $2.07 \pm 1.70$ & $2.06 \pm 1.10$ & 0.714 \\
\hline HDL (mmol/l) & $1.04 \pm 0.18$ & $1.04 \pm 0.21$ & $0.94 \pm 0.28$ & $1.05 \pm 0.24$ & 0.267 \\
\hline LDL (mmol/l) & $2.85 \pm 0.82$ & $2.55 \pm 0.78$ & $2.65 \pm 0.75$ & $2.73 \pm 0.96$ & 0.674 \\
\hline CRP (mg/l) & $8.87(5.47-15.91)$ & $18.11(7.71-34.44)$ & $13.32(4.22-21.06)$ & $10.05(4.73-18.64)$ & 0.922 \\
\hline MCP-1 (pg/ml) & $174.8(83.2-223.3)$ & $199.2(70.3-297.3)$ & $188.6(84.1-253.2)$ & $179.5(65.9-241.6)$ & 0.476 \\
\hline
\end{tabular}

RSG, rosiglitazone; BEZ, bezafibrate; BMI, body mass index; FBG, fasting blood glucose; Fins, fasting insulin; HOMA-IR, insulin resistance by homeostasis model assessment; HbA1c, hemoglobin A1c; TC, total cholesterol; TG, triglyceride; HDL, high-density lipoprotein; LDL, low-density lipoprotein; CRP, C-reactive protein; MCP-1, monocyte chemoattractant protein-1.

A

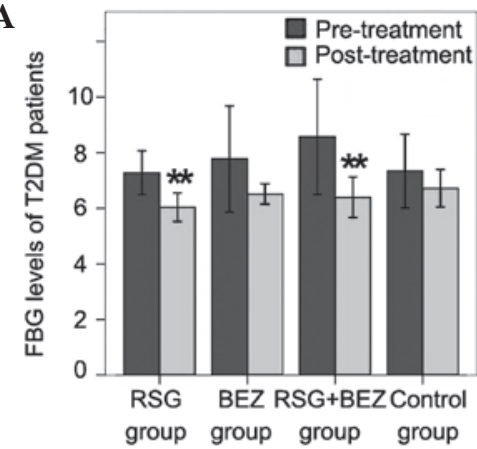

C

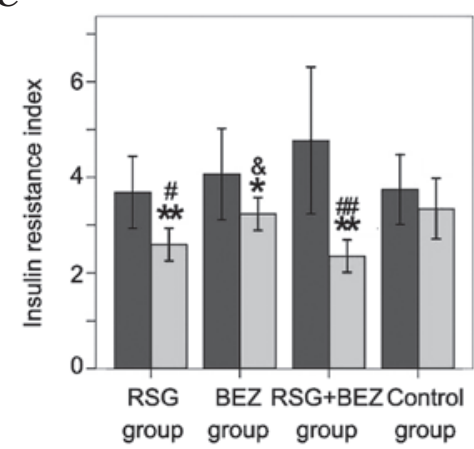

B

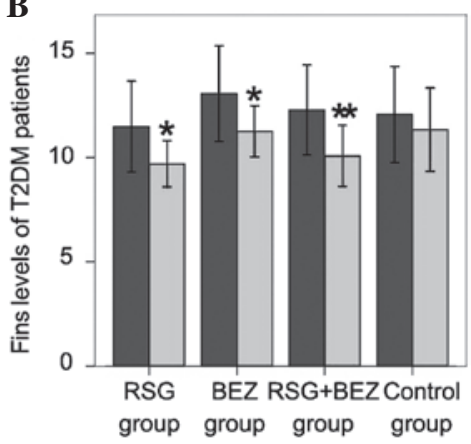

D

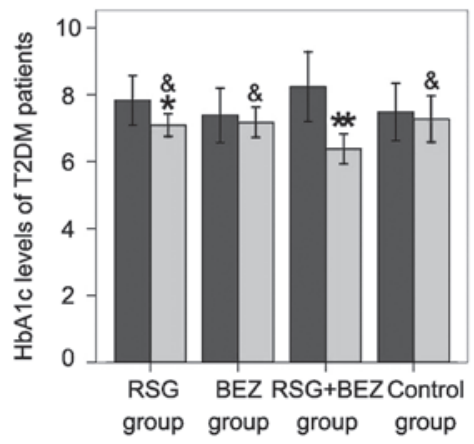

Figure 1. Blood glucose related parameters. (A) FBG, (B) Fins, (C) HOMA-IR and (D) HbAlc levels were determined before and after treatment. Each bar represents the mean \pm standard deviation $(\mathrm{SD} ; \mathrm{n}=20)$. ${ }^{*} \mathrm{P}<0.05,{ }^{* *} \mathrm{P}<0.01$, compared with pre-treatment in the same group; ${ }^{\star} \mathrm{P}<0.05$, compared with the combination group; ${ }^{\text {P }}<0.05,{ }^{\# \#} \mathrm{P}<0.01$, compared with the control group. FBG, fasting blood glucose; T2DM, type 2 diabetes mellitus; RSG, rosiglitazone; BEZ, bezafibrate; FI, fasting insulin; HbA1c, hemoglobin A1c; HOMA-IR, insulin resistance by homeostasis model assessment.

significant difference was detected. Patients in the RSG, BEZ and combination groups demonstrated improved HOMA-IR after 12 weeks of treatment; however, the amelioration was not significant in the control group (Fig. 1C). HOMA-IR in the RSG and combination groups was lower than that in the BEZ and control groups at 12 weeks and no significant difference was observed between the RSG group and the BEZ group. All patient HbAlc was not well-controlled before 
A

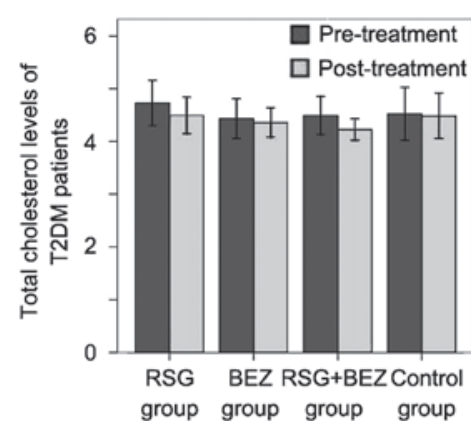

C

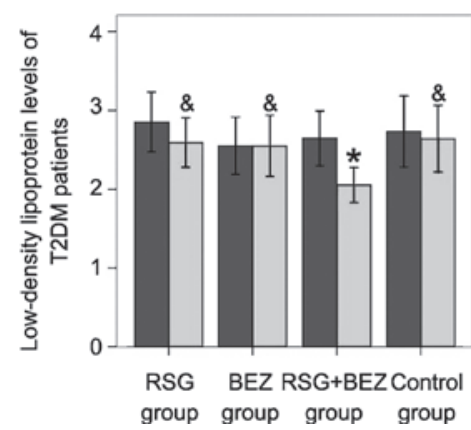

B

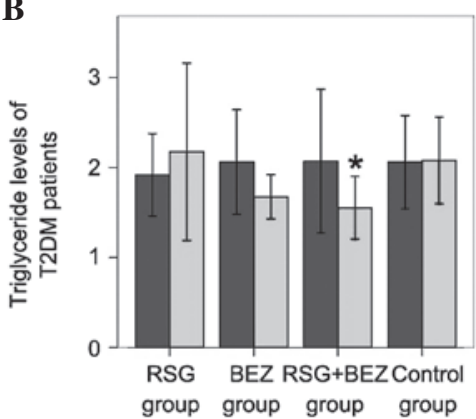

D

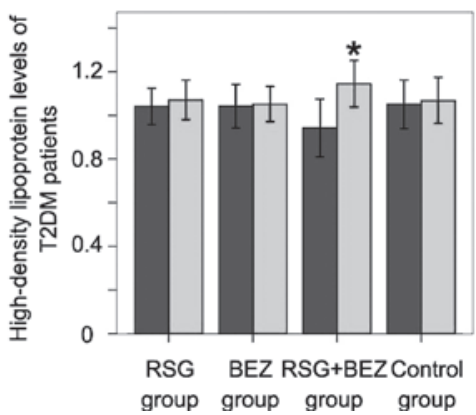

Figure 2. Changes in lipid parameters. (A) TC, (B) TG, (C) LDL and (D) HDL levels were determined before and after treatment. Each bar represents the mean \pm standard deviation $(\mathrm{SD} ; \mathrm{n}=20)$. ${ }^{*} \mathrm{P}<0.05$, compared with pre-treatment in the same group; ${ }^{\circledR} \mathrm{P}<0.05$, compared with the combination group. T2DM, type 2 diabetes mellitus; RSG, rosiglitazone; BEZ, bezafibrate; TC, total cholesterol; TG, triglyceride; LDL, low-density lipoprotein; HDL, high-density lipoprotein.

A

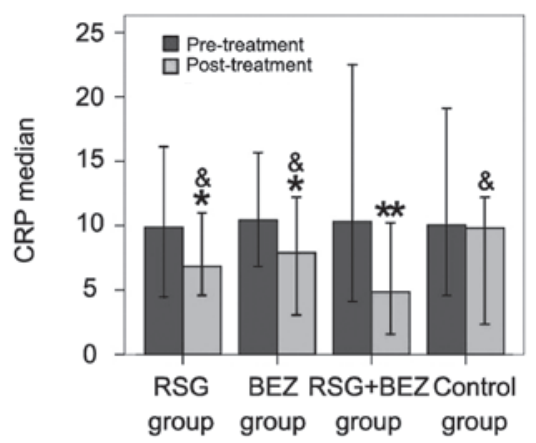

B

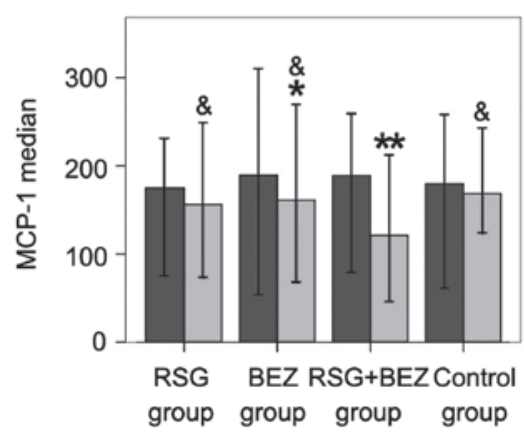

Figure 3. Plasma inflammatory cytokine levels in T2DM patients. (A) CRP and (B) MCP-1 levels were determined before and after treatment. Each bar represents the median and the range $(\mathrm{n}=20) .{ }^{*} \mathrm{P}<0.05,{ }^{* * *} \mathrm{P}<0.01$, compared with pre-treatment in the same group; ${ }^{\&} \mathrm{P}<0.05$, compared with the combination group. CRP, C-reactive protein; RSG, rosiglitazone; BEZ, bezafibrate; MCP-1, monocyte chemoattractant protein-1; T2DM, type 2 diabetes mellitus.

treatment (Fig. 1D). With RSG and combined treatment for 12 weeks, HbAlc markedly decreased compared with that before treatment; however, the decreases were not significant in the BEZ and control groups. HbAlc in the combination group was almost reduced to a normal level ( $\mathrm{HbA1c}<7 \%)$.

Changes in lipid parameters. After 12 weeks treatment, total cholesterol (TC) levels in the four groups all decreased; however, no significant differences were observed compared with those before treatment (Fig. 2A). TG levels in the RSG and control groups were slightly increased without significant differences (Fig. 2B), while in the BEZ and combination groups, TG was markedly decreased. The low-density lipoprotein (LDL) level in the combination group was markedly decreased (Fig. 2C); however, the reduction of HDL levels in the other groups was not significant. After 12 weeks of treatment, the HDL level in the combination group was markedly increased, while the HDL levels in the other groups were not significantly elevated (Fig. 2D).

Plasma CRP and MCP-1 levels. After 12 weeks of treatment, the CRP level in each of the four groups decreased (Fig. 3A). Significant differences in CRP and MCP-1 levels before and after treatment were observed in the RSG, BEZ and combination groups; however, this was not observed in the control group (Fig. 3A and B). Moreover, the levels of CRP and MCP-1 in the combination group decreased more than those in the other treatment groups.

Chemokine secretion from isolated monocytes in response to LPS. To test whether monocytes isolated from T2DM patients with CAD demonstrate an enhanced inflammatory response, PBMCs were incubated with LPS $(0.01-0.1 \mu \mathrm{g} / \mathrm{ml})$ for $24 \mathrm{~h}$. LPS at $0.01 \mathrm{lg} / \mathrm{ml}$ induced greater MCP-1 secretion by PBMCs from T2DM patients with CAD than from 


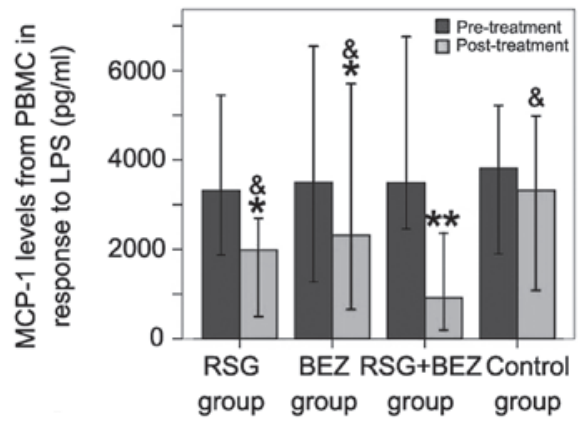

Figure 4. Effect of combined RSG and BEZ on MCP-1 levels secreted by PBMCs in response to LPS. PBMCs in T2DM patients with CAD were incubated with LPS for $24 \mathrm{~h}$. Then, the supernatant was harvested and MCP-1 levels were determined by ELISA. Each bar represents the mean \pm standard deviation (SD). ${ }^{*} \mathrm{P}<0.05,{ }^{* *} \mathrm{P}<0.01$, compared with pre-treatment in the same group; ${ }^{\&} \mathrm{P}<0.05$, compared with the combination group. MCP-1, monocyte chemoattractant protein 1; PBMC, peripheral blood mononuclear cell; LPS, lipopolysaccharide; RSG, rosiglitazone; BEZ, bezafibrate; T2DM, type 2 diabetes mellitus; CAD, coronary artery disease; ELISA, enzyme-linked immunosorbent assay.

control subjects (Fig. 4). RSG, BEZ and combined RSG and BEZ treatment for 12 weeks significantly decreased low-dose LPS-induced MCP-1 secretion. Combined treatment with RSG and BEZ inhibited the LPS-induced MCP-1 secretion more efficiently than RSG or BEZ used alone.

\section{Discussion}

T2DM is an independent risk factor of CAD. Epidemiological investigation revealed that patients with T2DM had a 2- to 4-fold higher risk than normal individuals of suffering from CAD (24). In the present study, we demonstrated that the combination of PPAR $\gamma$ and PPAR $\alpha$ agonists downregulates the blood glucose and lipid levels more efficiently in T2DM patients with CAD than the PPAR $\gamma$ or PPAR $\alpha$ agonist alone. The combination of PPAR $\gamma$ and PPAR $\alpha$ agonists also inhibited inflammatory cytokine secretion in the T2DM patients with CAD and attenuated the LPS-induced MCP-1 secretion by PBMCs in the T2DM patients. These results suggest that the combination of RSG and BEZ is more effective than RSG or BEZ alone for T2DM patients with CAD, and acts by inhibiting the secretion of inflammatory cytokines from monocytes.

RSG, a well-recognized oral anti-diabetic drug, belongs to the thiazolidinedione class of drugs. RSG increases the expression of glucose transporter-1 and facilitates the uptake of glucose by muscle and fat tissue through its receptors. Thereby, it reduces the output of liver glucose and blood glucose and improves hyperinsulinemia $(25,26)$. RSG also acts as an insulin sensitizer. It inhibits glucagon production and thus increases insulin sensitivity. In the present study, we demonstrated that RSG is capable of alleviating IR and decreasing FBG and HbA1c in T2DM patients. Similar results were observed in the BEZ group. However, when treated with combined RSG and BEZ for 12 weeks, the FBG, HbA1c, insulin and HOMA-IR all reached satisfactory levels. The results indicate that the combination of PPAR $\alpha / \gamma$ agonists improves glucose metabolism more effectively than the PPAR $\gamma$ or PPAR $\alpha$ agonist alone.

T2DM patients usually have one or more lipid abnormalities. The main characteristics of dyslipidemia in T2DM patients are an elevated TG level, increased LDL level and decreased HDL level. LDL cholesterol is a major risk factor for CVD in general and in particular in T2DM patients. Statins are extremely effective drugs for reducing the levels of LDL cholesterol. Numerous clinical trials have shown that treatment with statins lowers the rates of heart attack and cardiovascular mortality. Moreover, fibrates reduce TGs and increase the blood levels of HDL cholesterol, which is considered as 'good' cholesterol. However, there has been no definitive evidence from previous clinical trials that have used combined fibrate and statin to control blood lipid levels and thereby reduce the risk of heart attack and stroke in T2DM patients. In the present study, we identified that after 12 weeks treatment with combined RSG and BEZ, the TC, TG, LDL-C and HDL-C levels significantly improved. However, no significant changes in the lipid parameters, were observed in the other groups suggesting that the combination of the two drugs produces an improved outcome compared with RSG or BEZ alone.

Chronic inflammation has been shown to be a common precursor of T2DM and CAD, while hyperglycemia and IR may contribute to inflammation. Therefore, inflammation may act as a bridge between T2DM and coronary atherosclerosis (27). The process of PBMC migration into the vascular intima and transformation to foam cells, constitutes the early event of atherosclerosis and is regulated by plasma inflammatory cytokines, including MCP-1 and CRP. MCP-1 is secreted mainly by endothelial cells, vascular smooth muscle cells, monocytes and macrophages. Elevation of the MCP-1 level in CAD patients often indicates the increase and activation of inflammatory cells in the plaque. The increase of MMPs degrades structural matrices in the fibrous cap, which causes plaque rupture and vessel injury. Therefore, MCP-1 plays an important role in the acute coronary syndrome caused by atherosclerosis and plaque rupture (28). PPARs negatively regulate $\mathrm{NF}-\kappa \mathrm{B}$ and stimulate protein-1 activity, thereby inhibiting inflammatory gene transcription (29). Studies in animal models verified that PPAR $\alpha$ and PPAR $\gamma$ are able to exert anti-atherosclerotic effects in vivo. Clinical studies suggested that PPAR $\alpha$ or PPAR $\gamma$ alone are capable of lowering plasma inflammatory cytokine secretion in T2DM patients with CAD (30-33). Our study revealed that RSG or BEZ alone was able to lower plasma CRP and MCP-1 levels in T2DM patients with CAD. However, the effect was clearer with combined RSG and BEZ, indicating that a combination of RSG and BEZ alleviates the inflammatory reaction more effectively. The mechanisms underlying the effect of RSG or BEZ on CRP and MCP-1 levels may include the modulation of $\mathrm{NF}-\kappa \mathrm{B}, \mathrm{AP}-1$ and signal transducer and activator of transcription (STAT) signal pathways. The present study demonstrated that a combination of RSG and BEZ exerts a more efficient anti-inflammatory effect than RSG or BEZ alone, indicating that a combination of PPAR $\alpha / \gamma$ agonists is capable of inhibiting the expression of MCP-1, thus delaying the process of atherosclerosis.

In conclusion, our study demonstrated that a combination of RSG and BEZ is capable of reducing the level of blood glucose, alleviating IR and improving lipid modulation. A combination of RSG and BEZ is also able to lower plasma CRP and MCP-1 levels and decrease low-dose LPS-induced MCP-1 secretion by PBMCs in T2DM patients. The effects 
of combined RSG and BEZ are greater than those of RSG or BEZ used alone. The results of the present study suggest that the combination of PPAR $\alpha / \gamma$ agonists may be more effective for the treatment of T2DM patients with CAD.

\section{References}

1. Haffner SM, Lehto S, Rönnemaa T, Pyörälä K and Laakso M: Mortality from coronary heart disease in subjects with type 2 diabetes and in nondiabetic subjects with and without prior myocardial infarction. N Engl J Med 339: 229-234, 1998.

2. Shirani J and Dilsizian V: Screening asymptomatic patients with type 2 diabetes mellitus for coronary artery disease: does it improve patient outcome? Curr Cardiol Rep 12: 140-146, 2010.

3. Desvergne B and Wahli W: Peroxisome proliferator-activated receptors: nuclear control of metabolism. Endocr Rev 20: 649-688, 1999

4. Becker J, Delayre-Orthez C, Frossard N and Pons F: Regulation of inflammation by PPARs: a future approach to treat lung inflammatory diseases? Fundam Clin Pharmacol 20: 429-447, 2006.

5. Calkin AC and Thomas MC: PPAR agonists and cardiovascular disease in diabetes. PPAR Res 2008: 245410, 2008.

6. Inoue I, Goto S, Mizotani K, et al: Lipophilic HMG-CoA reductase inhibitor has an anti-inflammatory effect: reduction of mRNA levels for interleukin-1beta, interleukin-6, cyclooxygenase-2, and $\mathrm{p} 22$ phox by regulation of peroxisome proliferator-activated receptor alpha (PPARalpha) in primary endothelial cells. Life Sci 67: 863-876, 2000

7. Staels B, Koenig W, Habib A, et al: Activation of human aortic smooth-muscle cells is inhibited by PPARalpha but not by PPARgamma activators. Nature 393: 790-793, 1998.

8. Turay J, Grniaková V and Valka J: Changes in paraoxonase and apolipoprotein A-I, B, C-III and E in subjects with combined familiar hyperlipoproteinemia treated with ciprofibrate. Drugs Exp Clin Res 26: 83-88, 2000.

9. Marx N, Sukhova G, Murphy C, Libby P and Plutzky J: Macrophages in human atheroma contain PPARgamma: differentiation-dependent peroxisomal proliferator-activated receptor gamma (PPARgamma) expression and reduction of MMP-9 activity through PPARgamma activation in mononuclear phagocytes in vitro. Am J Pathol 153: 17-23, 1998.

10. Jiang C, Ting AT and Seed B: PPAR-gamma agonists inhibit production of monocyte inflammatory cytokines. Nature 391 : 82-86, 1998.

11. Delerive P, De Bosscher K, Besnard S, et al: Peroxisome proliferator-activated receptor alpha negatively regulates the vascular inflammatory gene response by negative cross-talk with transcription factors NF-kappaB and AP-1. J Biol Chem 274: 32048-32054, 1999.

12. Chakrabarti R, Vikramadithyan RK, Misra P, et al: Ragaglitazar: a novel PPAR alpha PPAR gamma agonist with potent lipid-lowering and insulin-sensitizing efficacy in animal models. Br J Pharmacol 140: 527-537, 2003.

13. Mittra S, Sangle G, Tandon R, et al: Increase in weight induced by muraglitazar, a dual PPARalpha/gamma agonist, in $\mathrm{db} / \mathrm{db}$ mice: adipogenesis/or oedema? Br J Pharmacol 150: 480-487, 2007.

14. Calkin AC, Allen TJ, Lassila M, Tikellis C, Jandeleit-Dahm KA and Thomas MC: Increased atherosclerosis following treatment with a dual PPAR agonist in the ApoE knockout mouse. Atherosclerosis 195: 17-22, 2007.

15. Calkin AC, Cooper ME, Jandeleit-Dahm KA and Allen TJ Gemfibrozil decreases atherosclerosis in experimental diabetes in association with a reduction in oxidative stress and inflammation. Diabetologia 49: 766-774, 2006.
16. Calkin AC, Forbes JM, Smith CM, et al: Rosiglitazone attenuates atherosclerosis in a model of insulin insufficiency independent of its metabolic effects. Arterioscler Thromb Vasc Biol 25: 1903-1909, 2005

17. Nissen SE, Wolski K and Topol EJ: Effect of muraglitazar on death and major adverse cardiovascular events in patients with type 2 diabetes mellitus. JAMA 294: 2581-2586, 2005.

18. Tordjman K, Bernal-Mizrachi C, Zemany L, et al: PPARalpha deficiency reduces insulin resistance and atherosclerosis in apoE-null mice. J Clin Invest 107: 1025-1034, 2001.

19. Mardones P, Pilon A, Bouly M, et al: Fibrates down-regulate hepatic scavenger receptor class B type I protein expression in mice. J Biol Chem 278: 7884-7890, 2003.

20. Berger J and Moller DE: The mechanisms of action of PPARs. Annu Rev Med 53: 409-435, 2002.

21. Saha SA, Kizhakepunnur LG, Bahekar A and Arora RR: The role of fibrates in the prevention of cardiovascular disease - a pooled meta-analysis of long-term randomized placebo-controlled clinical trials. Am Heart J 154: 943-953, 2007.

22. Zeng X, Dai J, Remick DG and Wang X: Homocysteine mediated expression and secretion of monocyte chemoattractant protein-1 and interleukin-8 in human monocytes. Circ Res 93: 311-320, 2003.

23. Matthews DR, Hosker JP, Rudenski AS, Naylor BA, Treacher DF and Turner RC: Homeostasis model assessment: insulin resistance and beta-cell function from fasting plasma glucose and insulin concentrations in man. Diabetologia 28: 412-419, 1985.

24. Laakso M: Hyperglycaemia and cardiovascular disease in type 2 diabetes. Diabetes 48: 937-942, 1999.

25. Tenenbaum A, Motro M, Fisman EZ, et al: Peroxisome proliferator-activated receptor ligand bezafibrate for prevention of type 2 diabetes mellitus in patients with coronary artery disease. Circulation 109: 2197-2202, 2004.

26. Jones IR, Swai A, Taylor R, Miller M, Laker MF and Alberti KG: Lowering of plasma glucose concentrations with bezafibrate in patients with moderately controlled NIDDM. Diabetes Care 13: 855-863, 1990 .

27. Sobel BE: Optimizing cardiovascular outcomes in diabetes mellitus. Am J Med 120 (9 Suppl 2): S3-S11, 2007.

28. Liuzzo G, Goronzy JJ, Yang H, et al: Monoclonal T-cell proliferation and plaque instability in acute coronary syndromes. Circulation 101: 2883-2888, 2000.

29. Chinetti G, Fruchart JC and Staels B: Peroxisome proliferatoractivated receptors (PPARs): nuclear receptors with functions in the vascular wall. Z Kardiol 90 (Suppl 3): 125-132, 2001.

30. Wang G, Wei J, Guan Y, Jin N, Mao J and Wang X: Peroxisome proliferator-activated receptor-gamma agonist rosiglitazone reduces clinical inflammatory responses in type 2 diabetes with coronary artery disease after coronary angioplasty. Metabolism 54: 590-597, 2005.

31. Wang TD, Chen WJ, Lin JW, Cheng CC, Chen MF and Lee YT: Efficacy of fenofibrate and simvastatin on endothelial function and inflammatory markers in patients with combined hyperlipidemia: relations with baseline lipid profiles. Atherosclerosis 170: 315-323, 2003.

32. Han SH, Quon MJ and Koh KK: Beneficial vascular and metabolic effects of peroxisome proliferator-activated receptoralpha activators. Hypertension 46: 1086-1092, 2005.

33. Mohanty P, Aljada A, Ghanim H, et al: Evidence for a potent antiinflammatory effect of rosiglitazone. J Clin Endocrinol Metab 89: 2728-2735, 2004. 